Original article

\title{
Long term outcomes of acute and transient psychotic disorders: The missed opportunity of preventive interventions
}

\author{
Grazia Rutigliano ${ }^{\mathrm{a}, \mathrm{b}, *}$, Sergio Merlino ${ }^{\mathrm{a}, \mathrm{b}}$, Amedeo Minichino $^{\mathrm{a}}$, Rashmi Patel ${ }^{\mathrm{a}}$, \\ Cathy Davies $^{\mathrm{a}}$, Dominic Oliver ${ }^{\mathrm{a}}$, Andrea De Micheli ${ }^{\mathrm{a}}$, Philip McGuire ${ }^{\mathrm{a}}$, \\ Paolo Fusar-Poli ${ }^{\mathrm{a}, \mathrm{c}, \mathrm{d}, \mathrm{e}}$
}

${ }^{a}$ Early Psychosis: Interventions and Clinical Detection (EPIC) Lab, Department of Psychosis Studies, Institute of Psychiatry, Psychology E Neuroscience, King's College London, 16 De Crespigny Park, SE5 8AF, London, United Kingdom

${ }^{\mathrm{b}}$ Department of Clinical and Experimental Medicine, University of Pisa, Via Roma, 67, 56126, Pisa, Italy

' OASIS Service, South London and Maudsley NHS Foundation Trust, 190 Kennington Ln, Lambeth, SE11, London, United Kingdom

${ }^{\mathrm{d}}$ National Institute for Health Research (NIHR) Maudsley Biomedical Research Centre, De Crespigny Park, Camberwell, SE5 8AF, London, United Kingdom

e Department of Brain and Behavioral Sciences, University of Pavia, Pavia, Italy

\section{A R T I C L E I N F O}

\section{Article history:}

Received 11 January 2018

Received in revised form 5 May 2018

Accepted 8 May 2018

Available online 19 May 2018

\section{Keywords:}

Acute and transient psychotic disorders

Brief psychotic disorders

Psychosis risk

Schizophrenia

Prevention

Early detection

\begin{abstract}
A B S T R A C T
Background: Acute and transient psychotic disorders (ATPD) are characterized by an acute onset and a remitting course, and overlap with subgroups of the clinical high-risk state for psychosis. The long-term course and outcomes of ATPD are not completely clear.

Methods: Electronic health record-based retrospective cohort study, including all patients who received a first index diagnosis of ATPD (F23, ICD-10) within the South London and Maudsley (SLaM) National Health Service Trust, between 1 st April 2006 and 15th June 2017. The primary outcome was risk of developing persistent psychotic disorders, defined as the development of any ICD-10 diagnoses of nonorganic psychotic disorders. Cumulative risk of psychosis onset was estimated through Kaplan-Meier failure functions (non-competing risks) and Greenwood confidence intervals.

Results: A total of 3074 patients receiving a first index diagnosis of ATPD (F23, ICD-10) within SLaM were included. The mean follow-up was 1495 days. After 8-year, 1883 cases (61.26\%) retained the index diagnosis of ATPD; the remaining developed psychosis. The cumulative incidence (Kaplan-Meier failure function) of risk of developing any ICD-10 non-organic psychotic disorder was $16.10 \%$ at 1 -year (95\%CI $14.83-17.47 \%$ ), $28.41 \%$ at 2-year (95\%CI 26.80-30.09\%), 33.96\% at 3-year (95\% CI 32.25-35.75\%), 36.85\% at 4-year (95\%CI $35.07-38.69 \%), 40.99 \%$ at 5-year (95\% CI 39.12-42.92\%), $42.58 \%$ at 6 -year (95\%CI 40.67-44.55\%), 44.65\% at 7year (95\% CI 42.66-46.69\%), and 46.25\% at 8-year (95\% CI 44.17-48.37\%). The cumulative risk of schizophrenia-spectrum disorder at 8-year was 36.14\% (95\% CI 34.09-38.27\%).

Conclusions: Individuals with ATPD have a very high risk of developing persistent psychotic disorders and may benefit from early detection and preventive treatments to improve their outcomes.
\end{abstract}

(C) 2018 Elsevier Masson SAS. All rights reserved.

\section{Introduction}

Accounts of brief and acute psychotic episodes [1] are found across different 19th- and 20th-century European psychiatric traditions under various terms (for a recent historical review of these construct see [2]). The unifying feature across these

\footnotetext{
* Corresponding author at: Early Psychosis: Interventions and Clinical Detection (EPIC) Lab, Department of Psychosis Studies, Institute of Psychiatry, Psychology \& Neuroscience, King's College London, 16 De Crespigny Park, SE5 8AF, London, United Kingdom.

E-mail address: grazia.rutigliano@kcl.ac.uk (G. Rutigliano).
}

constructs is of short-lived psychotic symptoms that remit within a relatively short period of time. In the modern taxonomy, brief psychotic episodes are classified under "acute and transient psychotic disorders" (ATPDs) in ICD-10, and "brief psychotic disorder" (BPD) in DSM-5 [3]. The World Health Organization defines ICD-10 ATPDs as psychotic episodes fully remitting within 1 to 3 months, with (a) acute onset within 2 weeks; (b) presence of typical syndromes; and (c) presence of associated acute stress [4]. Specifically, the ATPDs include six subtypes: acute polymorphic psychotic disorder without symptoms of schizophrenia (F23.0, remission within 3 months), acute polymorphic psychotic disorder with schizophrenic symptoms (F23.1, remission within 1 month); acute schizophrenia-like psychotic disorder (F23.2, remission 
within 1 month); acute predominantly delusional psychotic disorder (F23.3, remission within 3 months); and 'other' (F23.8) and 'unspecified' (F23.9) acute and transient psychotic disorders. Complete remission within 1 or 3 months sets the ATPDs with schizophrenic symptoms apart from schizophrenia (the ICD-10 diagnosis of schizophrenia requires at least 1 month's duration), and the ATPDs with polymorphic or delusional features apart from persistent delusional disorder (which lasts longer than 3 months) [3]. Typical syndromes differ across the 6 subtypes, from a "polymorphic" rapidly changing and variable state, which encompasses emotional turmoil, perplexity, and alterations in motility (F23.0 Acute polymorphic psychotic disorder without symptoms of schizophrenia), to subtypes including typical schizophrenic symptoms (F23.1 Acute polymorphic psychotic disorder with symptoms of schizophrenia; F23.2 Acute schizophrenia-like psychotic disorder; F23.3 Other acute predominantly delusional psychotic disorders). Finally, a character may be used to indicate whether the first psychotic symptoms emerged within around 2 weeks of one or more events that "would be regarded as stressful to most people in similar circumstances, within the culture of the person concerned". Examples of such events include bereavement, marriage, terrorism and unexpected loss of partner or job, but should not include long-standing difficulties.

Conversely, in the Diagnostic and Statistical Manual of Mental Disorders, fifth edition (DSM-5), the American Psychiatric Association classifies Brief Psychotic Disorders (BPD) as psychotic conditions lasting 1 day or more but less than 1 month, with complete remission to the premorbid level of functioning [5]. A diagnostic comparison of these two categories has been presented in a recent publication by our group, which is beyond the scope of the current study [2].

Clinical research interest in ATPDs has grown substantially since their reconceptualization as a state of risk for the development of persistent psychotic disorders, approximately two decades ago. Accordingly, the Clinical High Risk State for Psychosis (CHR-P) [6] defines individuals who are at risk of developing psychotic disorders but not other emerging mental disorders [7,8]. The CHR-P group includes the Brief and Limited Intermittent Psychotic Symptoms (BLIPS) subgroup, which also features short-lived psychotic episodes [9]. Compared to ATPDs -which are defined clinically-, the BLIPS are defined through specific psychometric tools [10]. There is substantial diagnostic (around 70\% of BLIPS cases also meet ATPD criteria [11]) and prognostic overlap (with similar levels of psychosis risk over time [2]) between the BLIPS and ATPD categories. Reconceptualization of ATPDs within the CHR-P framework has allowed early detection and preventive treatments to be offered to some of these patients [12].

Whatever the designation, there is converging evidence that brief psychotic episodes are associated with a very high risk of developing persistent psychotic disorders, in particular schizophrenia spectrum psychoses. Recently, we have meta-analytically estimated that up to half of patients $(0.56,95 \%$ CI $0.52-0.60)$ with an ATPD (and BPD) developed another psychotic disorder at an average follow-up of 4.5 years, mostly schizophrenia-spectrum disorders (encompassing schizophrenia 0.21 95\% CI 0.16-0.25, schizophreniform disorder $0.0295 \%$ CI $0.00-0.14$, and schizoaffective disorder $0.0295 \% \mathrm{CI} 0.00-0.06$ ), and less frequently affective psychoses $0.1295 \%$ CI $0.07-0.16$ [13]. There is less evidence on the longer-term outcomes of ATPD individuals. Our meta-analysis uncovered 8 cohort studies reporting on ATPD outcomes at 8 years or longer [2]. However, the vast majority of them were based on small samples, ranging from 15 to 54 patients [14-20], with inaccurate outcome estimates. Only one study involved a large cohort of ATPD cases [21]. Since the long-term results of this study have not been validated externally (outside the
Danish psychiatric population), their generalizability is currently unclear.

The current study sought to overcome this limitation in knowledge. Here, we report the longitudinal diagnostic outcomes of ATPDs as recorded in a clinical case register that was representative of secondary mental health care in the UK. Our first aim was to report and describe the risk of developing persistent psychotic disorders in the long term, and then to report the specific risk of developing schizophrenia-spectrum disorders. As additional analyses, we also described all diagnostic changes occurring within the follow-up period.

\section{Materials and methods}

\subsection{Data source}

Data for this study were automatically extracted from the South London and Maudsley (SLaM) Biomedical Research Centre (BRC) Case Register, using the Clinical Record Interactive Search tool (CRIS). Developed in 2008, CRIS enables the search of anonymized, real-time information from the electronic health records of patients receiving treatment within SLaM [22]. SLaM is a large National Health Service (NHS) Trust providing specialist mental health care to a catchment area of 1.3 million residents across four South London boroughs (Lambeth, Southwark, Lewisham and Croydon) [22]. Every patient within SLaM has their full personal electronic health record, which must be continually updated by SLaM health care professionals as a legal requirement [22]. Since CRIS derives direct information from these electronic health records, it is an advantageous source of "real-world" and "real-time" information on routine mental health care [23]. CRIS was approved by the Oxfordshire Research Ethics Committee C (reference 08/H0606/71+5). Access to the anonymized data for this study was reviewed, monitored and audited by an Oversight Committee, which carries representation from the SLaM Cauldicott Guardian and is chaired by a service user. The CRIS Oversight Committee is responsible for ensuring that all research applications comply with ethical and legal guidelines; approval for this study was granted on the 4th August 2014. CRIS has been extensively used in over 70 previous studies, some of which appeared in high impact factor journals [24-26].

\subsection{Study population}

All individuals who received a first index diagnosis of ATPD (F23, ICD-10) within SLaM between 1st April 2006 and 15th June 2017 were initially considered eligible. Consent from patients was not needed as data are completely de-identified [22]. We then excluded those with very short-term diagnostic instability, defined as diagnostic change occurring within the 3 months immediately following the first index diagnosis of ATPD (i.e., in the context of the index episode itself). This was intended as a more robust and complete method of measuring diagnoses, excluding provisional highly unstable diagnoses recorded during the period of observation. This approach has already been validated in a previous study by our group, published in a high impact factor journal [27]. The remaining sample was therefore composed of all patients with a first index diagnosis of ATPD, who retained the index diagnosis up to 3 months.

\subsection{Study measures}

The primary outcome was the long-term (up to 8-year) risk of developing persistent psychotic disorders, defined as the onset of the first ICD-10 diagnoses of non-organic psychotic disorders since index diagnosis: schizophrenia-spectrum disorders (schizophrenia [F20.X, 
except F20.4/F20.5], schizoaffective disorder [F25.X], delusional disorders [F22.x, F24]); affective-spectrum psychoses (mania with psychotic symptoms [F30.2], bipolar affective disorder with psychotic symptoms [F31.2, F31.5], and depression with psychotic symptoms [F32.3/F33.3]); psychotic disorders due to psychoactive substance use ([F10-F19].5); unspecified non-organic psychoses (F28/F29); and severe mental and behavioural disorders associated with the puerperium (puerperal psychosis NOS, F53.1). To better clarify the course of ATPDs, we further provided a descriptive analysis of diagnostic changes following the first onset of persistent psychotic disorders.

Time to persistent psychotic disorders was assessed from the date of first index diagnosis of ATPD, censored at 15th June 2017.

\subsection{Statistical analysis}

This electronic health record-based cohort study was performed according to the REporting of Studies Conducted using Observational Routinely-collected health Data statement (RECORD) [28] (eTable 1).

We described baseline sociodemographic and clinical characteristics of the sample by mean and standard deviation (SD) for continuous variables, and by absolute and relative frequencies for categorical variables. Sociodemographic and clinical characteristics were then stratified across ATPD subgroups, using one-way ANOVA and Tukey post-hoc tests for continuous variables, and chi square tests for categorical variables. For descriptive purposes, we also obtained absolute and relative frequencies of every ICD-10 diagnosis of non-organic psychotic disorders at seven time-points $(6,12,18,24,48,72$, and 96 months).

The cumulative incidence of non-organic ICD-10 persistent psychotic disorders from ATPD during the follow-up period was estimated with Kaplan-Meier failure function [29] and Greenwood 95\% confidence intervals [30]. In a subsequent analysis, we specifically focused on the risk of developing schizophreniaspectrum disorders. Furthermore, we plotted Kaplan-Meier failure curves separately for ATPD subgroups with (F23.1 and F23.2) or without (F23.0, F23.3, F23.8, and F23.9) schizophrenic symptoms.

All analyses were conducted in STATA 12 (STATA Corp., TX, USA).

\section{Results}

\subsection{Sociodemographic and clinical characteristics of the sample}

Between 1st April 2006 and 15th June 2017, a total of 3286 subjects received a first index primary diagnosis of ATPD (F23, ICD-10) within the SLaM NHS Trust. After 3 months, 3074 subjects had retained the index ATPD diagnosis. Table 1 depicts the sociodemographic and clinical characteristics of these remaining 3074 patients. The mean age was 33.84 years $(S D=13.99)$ and $53 \%$ were male $(n=1633)$. Females were older than males, mean age 35.32 years $(S D=14.72)$ vs 32.53 years ( $S D=13.17)$. The majority were of black ethnicity ( $n=1353,45 \%)$, followed by white ethnicity $(n=1089,37 \%)$. The most frequent ATPD subtypes were unspecified non-organic psychoses (F23.8 Other acute and transient psychotic disorders (7.12\%), and F23.9 Acute and transient psychotic disorders unspecified (63.44\%)), together accounting for $71 \%(n=2169)$ of the total sample. Other subtypes were distributed as follows: F23.1 Acute polymorphic disorder with symptoms of schizophrenia, 5.82\% ( $n=179$ ); F23.2 Acute schizophrenia-like psychotic disorder, $14.02 \%(n=431)$; F.23.0 Acute polymorphic disorder without symptoms of schizophrenia, 4.78\% ( $\mathrm{n}=147)$; and F23.3 Other acute predominantly delusional psychotic disorders, 4.81\% $(n=148)$. ATPDs with recurrent course were relatively rare during the follow-up period ( $n=607,20 \%$ ). Approximately one-third of episodes of
Table 1

Sociodemographic and clinical characteristics of patients with a first index diagnosis of ATPD

\begin{tabular}{|c|c|c|c|c|}
\hline \multicolumn{5}{|l|}{ Sociodemographic characteristics } \\
\hline & & $\mathrm{N}$ & Mean & SD \\
\hline \multirow[t]{2}{*}{ Age } & & 3072 & 33.84 & 13.99 \\
\hline & & $\mathrm{N}$ & Count & $\%$ \\
\hline \multirow[t]{3}{*}{ Gender } & & 3074 & & \\
\hline & Males & & 1633 & 53.12 \\
\hline & Females & & 1441 & 46.88 \\
\hline \multirow[t]{6}{*}{ Ethnicity } & & 2981 & & \\
\hline & White & & 1089 & 36.53 \\
\hline & Black & & 1353 & 45.39 \\
\hline & Asian & & 232 & 7.78 \\
\hline & Mixed & & 70 & 2.35 \\
\hline & Other & & 237 & 7.95 \\
\hline \multicolumn{5}{|l|}{ Clinical characteristics } \\
\hline \multirow[t]{6}{*}{ ATPD subtype } & & 3074 & & \\
\hline & F23.0 & & 147 & 4.78 \\
\hline & F23.1 & & 179 & 5.82 \\
\hline & $\mathrm{F} 23.2$ & & 431 & 14.02 \\
\hline & F23.3 & & 148 & 4.81 \\
\hline & F23.8 or F23.9 & & 2169 & 70.56 \\
\hline \multirow[t]{3}{*}{ ATPD course } & & 3074 & & \\
\hline & Episodic & & 2467 & 80.25 \\
\hline & Recurrent & & 607 & 19.75 \\
\hline \multirow[t]{3}{*}{ Non-rapidly remitting symptoms } & & 3074 & & \\
\hline & $<30$ days & & 2092 & 68.05 \\
\hline & $>30$ days & & 982 & 31.95 \\
\hline \multirow[t]{3}{*}{ Associated Acute stress } & & 3074 & & \\
\hline & No & & 3040 & 98.89 \\
\hline & Yes & & 34 & 1.11 \\
\hline \multirow[t]{3}{*}{ Antipsychotics } & & 3074 & & \\
\hline & No & & 1076 & 35 \\
\hline & Yes & & 1998 & 65 \\
\hline
\end{tabular}

ATPD lasted more than 30 days ( $n=982,32 \%)$. An acute stress preceding ATPD was present in a small proportion of patients $(n=34,1 \%)$. Antipsychotics were offered to $65 \%(n=1998)$ of patients over the follow-up period.

\subsection{Sociodemographic and clinical differences across ATPD subtypes}

Patients with acute predominantly delusional psychotic disorders (F23.3) were comparatively older at ATPD onset relative to other ATPD subtypes (mean $=41.74, \mathrm{SD}=16.85$ ) (Table 2 ). In our sample, ATPD subtypes showed overlapping distributions in terms of gender $(\chi 2=7.68, \mathrm{p}=0.053)$ and ethnicity $\left(\chi^{2}=16.32, \mathrm{p}=0.177\right)$. ATPD subtypes with symptoms of schizophrenia had more frequent recurrent course $(\chi 2=33.25, \mathrm{p}<0.001)$ and longer duration $(\chi 2=19.24, \mathrm{p}<0.001)$. We did not observe any association between ATPD subtypes and associated acute stress $(\chi 2=4.78, \mathrm{p}=0.189)$ (Table 2).

\subsection{Risk of developing persistent psychotic disorders from ATPDs}

The mean follow-up was 1495 days (range: 48-4091). The mean time to psychosis transition was 661 days, with the first transition to psychosis observed at 91 days and the last at 2907 days. There were 1191 events, with the last onset of persistent psychotic disorders occurring at day 2907 (i.e., at 7.96 years), when 519 patients were still at risk. The cumulative incidence (Kaplan-Meier failure function) of risk of developing any ICD-10 non-organic psychotic disorder was $16.10 \%$ at 1 -year (95\%CI $14.83-17.47 \%$ ), $28.41 \%$ at 2 -year (95\%CI $26.80-30.09 \%$ ), $33.96 \%$ at 3 -year (95\% CI $32.25-35.75 \%), 36.85 \%$ at 4 -year $(95 \% \mathrm{CI}$ $35.07-38.69 \%$ ), $40.99 \%$ at 5 -year (95\% CI $39.12-42.92 \%), 42.58 \%$ at 6 -year (95\%CI $40.67-44.55 \%$ ), $44.65 \%$ at 7 -year (95\% CI $42.66-$ $46.69 \%$ ), and $46.25 \%$ at 8 -year (95\% CI $44.17-48.37 \%$ ) (Fig. 1). 
Table 2

Sociodemographic and clinical differences across ATPD subtypes.

\begin{tabular}{|c|c|c|c|c|c|c|c|c|c|c|}
\hline & \multicolumn{2}{|l|}{ F23.0 } & \multicolumn{2}{|c|}{ F23.1 or F23.2 } & \multicolumn{2}{|l|}{ F23.3 } & \multicolumn{2}{|c|}{ F23.8 or F23.9 } & \multirow[b]{2}{*}{$\mathrm{F}$} & \multirow[b]{2}{*}{$\mathrm{p}$} \\
\hline & Mean & SD & Mean & SD & Mean & SD & Mean & SD & & \\
\hline Age & 34.32 & 13.44 & 32.20 & 13.02 & 41.74 & 16.85 & 33.73 & 13.90 & 18.99 & $<0.001$ \\
\hline & Count & $\%$ & Count & $\%$ & Count & $\%$ & Count & $\%$ & $x^{2}$ & $\mathrm{P}$ \\
\hline Gender & & & & & & & & & 7.68 & 0.053 \\
\hline Males & 62 & 3.80 & 331 & 20.27 & 77 & 4.72 & 1163 & 71.22 & & \\
\hline Females & 85 & 5.90 & 279 & 19.36 & 71 & 4.93 & 1006 & 69.81 & & \\
\hline Ethnicity & & & & & & & & & 16.32 & 0.177 \\
\hline White & 48 & 4.41 & 210 & 19.28 & 58 & 5.33 & 773 & 70.98 & & \\
\hline Black & 62 & 4.58 & 288 & 21.29 & 51 & 3.77 & 952 & 70.36 & & \\
\hline Asian & 14 & 6.03 & 39 & 16.81 & 15 & 6.47 & 164 & 70.69 & & \\
\hline Mixed & 3 & 4.29 & 6 & 8.57 & 4 & 5.71 & 57 & 81.43 & & \\
\hline Other & 14 & 5.91 & 49 & 20.68 & 14 & 5.91 & 160 & 67.51 & & \\
\hline ATPD course & & & & & & & & & 33.25 & $<0.001$ \\
\hline Episodic & 122 & 4.95 & 439 & 17.79 & 124 & 5.03 & 1782 & 72.23 & & \\
\hline Recurrent & 25 & 4.12 & 171 & 28.17 & 24 & 3.95 & 387 & 63.76 & & \\
\hline Non-rapidly remitting symptoms & & & & & & & & & 19.24 & $<0.001$ \\
\hline$<30$ days & 87 & 4.16 & 380 & 18.16 & 104 & 4.97 & 1521 & 72.71 & & \\
\hline$>30$ days & 60 & 6.11 & 230 & 23.42 & 44 & 4.48 & 648 & 65.99 & & \\
\hline Associated Acute stress & & & & & & & & & 4.78 & 0.189 \\
\hline No & 146 & 4.80 & 599 & 19.70 & 148 & 4.87 & 2147 & 70.63 & & \\
\hline Yes & 1 & 2.94 & 11 & 32.35 & 0 & 0 & 22 & 64.71 & & \\
\hline
\end{tabular}

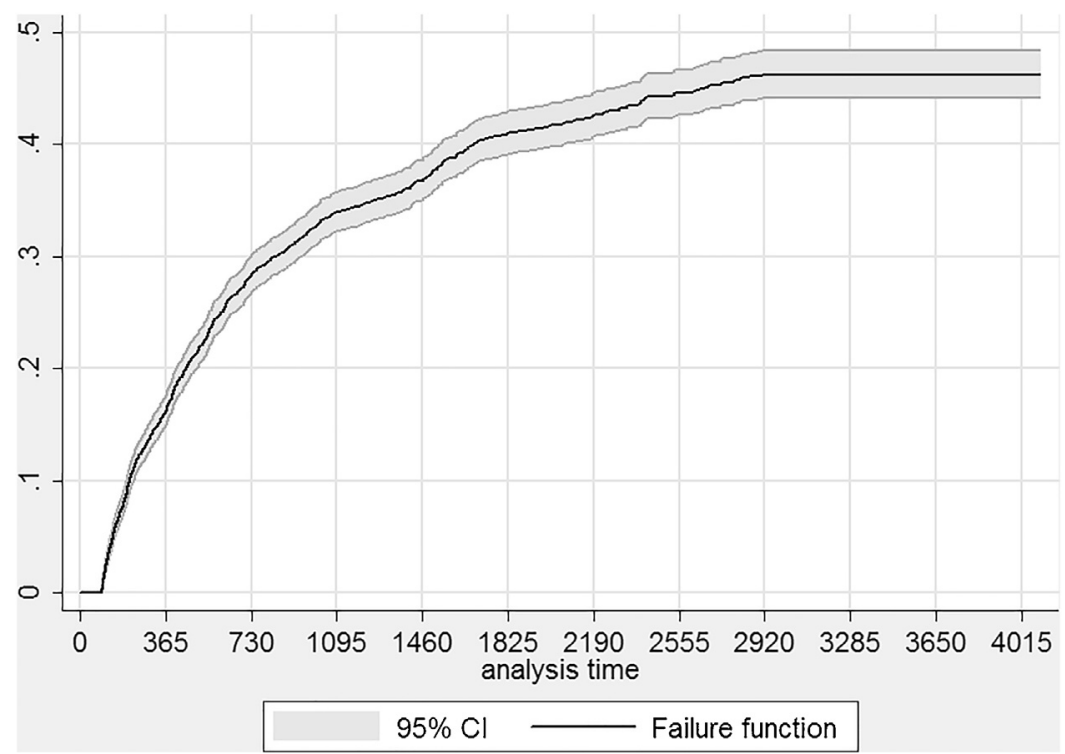

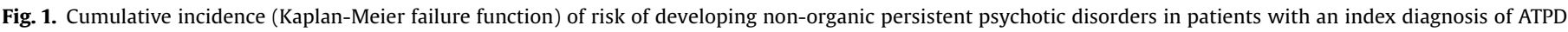

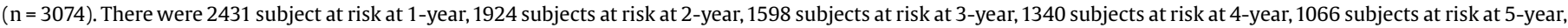
862 subjects at risk at 6-year, 655 subjects at risk at 7-year, and 515 subjects at risk at 8-year follow-up.

Fig. 2 depicts the diagnostic changes from a first index diagnosis of ATPD to any non-organic psychotic disorders -and individual spectra of psychotic disorders- across seven time points $(6,12,18$, $24,48,72$, and 96 months). As indicated, at 96 months 875 had received a diagnosis of schizophrenia spectrum disorders (726 of schizophrenia, 106 of schizoaffective disorders, and 43 of delusional disorders), 145 of affective-spectrum psychoses, 22 of psychotic disorders due to psychoactive substance use, 148 of unspecified non-organic psychoses, and 1 of severe mental and behavioural disorders associated with the puerperium [Puerperal psychosis NOS] (F53.1). Fig. 3 represents the cumulative incidence (Kaplan-Meier failure function) of risk of developing any ICD-10 non-organic persistent psychotic disorder, stratified for the presence or absence of schizophrenic symptoms during the index episode of ATPD.

\subsection{Risk of developing schizophrenia-spectrum disorders from ATPDs}

The mean time to transition to schizophrenia-spectrum disorders was 641 days, with the first transition observed at 91 days and the last at 2907 days. There were 875 events, with the last onset of schizophrenia-spectrum disorders occurring at day 2907 (i.e., at 7.96 years), when 519 patients were still at risk. The cumulative incidence (Kaplan-Meier failure function) of risk of developing schizophrenia-spectrum disorders was $12.42 \%$ at 1 -year (95\%CI $11.28-13.67 \%), 21.99 \%$ at 2 -year (95\%CI $20.50-23.58 \%$ ), $26.27 \%$ at 3-year (95\% CI 24.65-27.99\%), $28.93 \%$ at 4 -year (95\%CI $27.21-30.72 \%), 32.37 \%$ at 5 -year $(95 \% \mathrm{CI}$ $30.53-34.28 \%$ ), $33.35 \%$ at 6 -year (95\%CI $31.48-35.31 \%), 34.92 \%$ at 7-year (95\% CI $32.95-36.96 \%)$, and $36.14 \%$ at 8 -year $(95 \% \mathrm{CI}$ 34.09-38.27\%) (Fig. 4). As a supplementary result, we plotted 


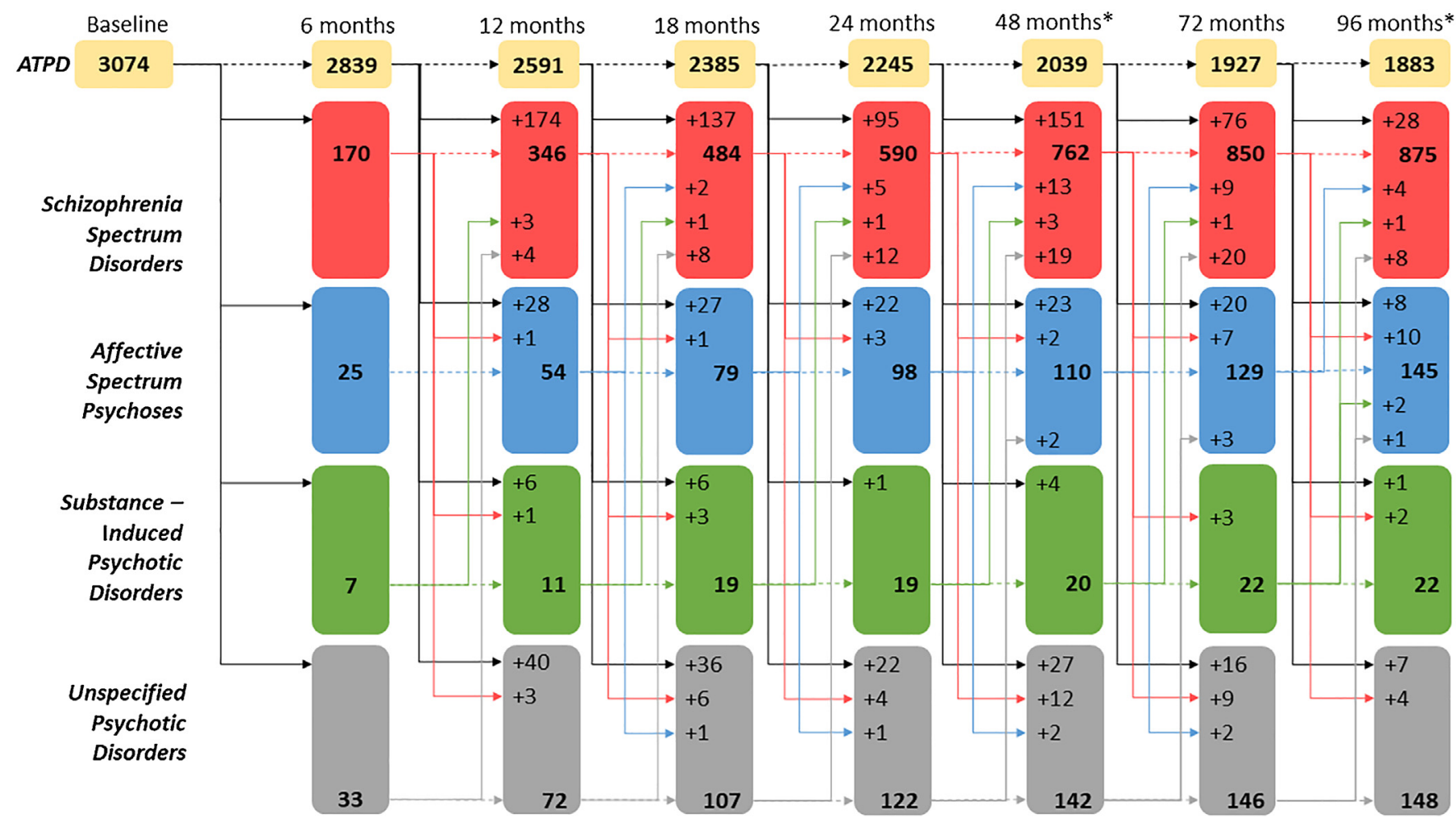

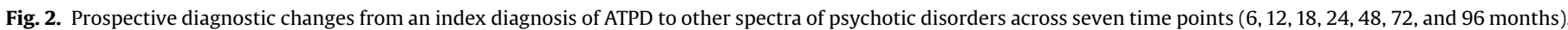

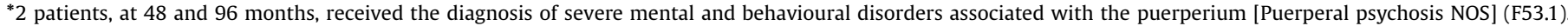

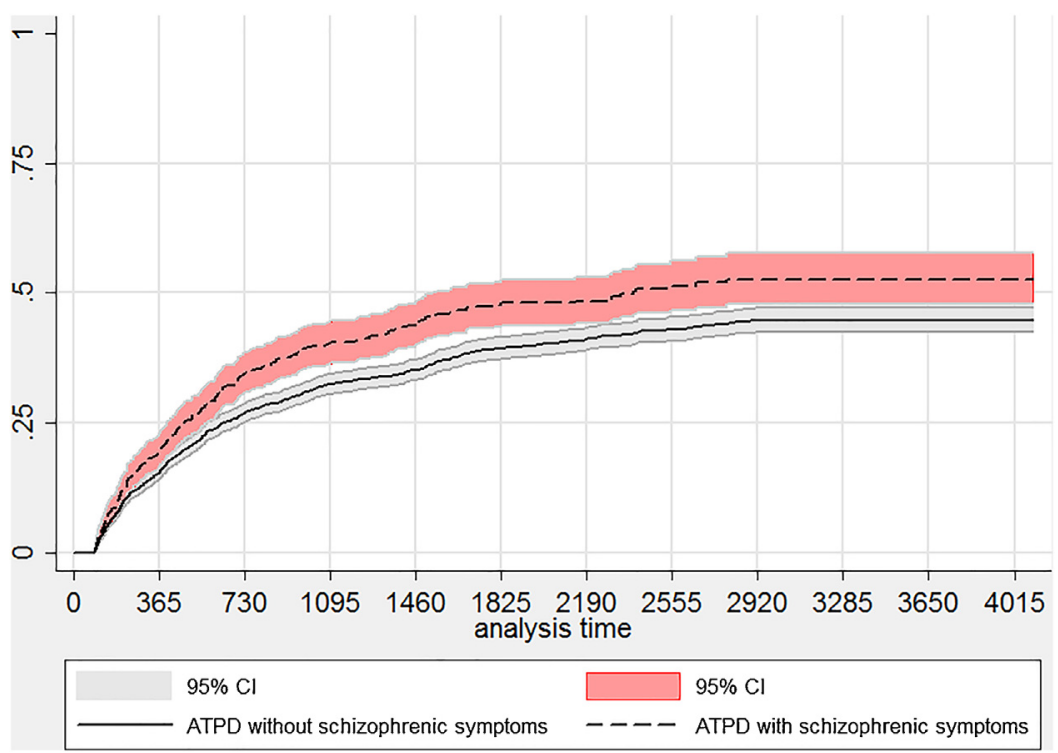

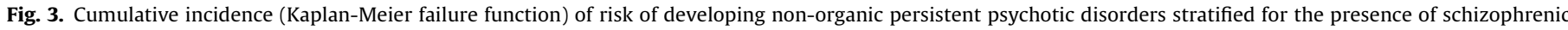
symptoms during the index ATPD episode.

the cumulative incidence (Kaplan-Meier failure function) of risk of developing schizophrenia-spectrum disorders stratified for the presence or absence of schizophrenic symptoms during the index episode of ATPD (eFig. 1).

\section{Discussion}

To the best of our knowledge, this is one of the few large-scale studies to report on the long-term outcomes of patients affected with ATPDs. A total of 3074 patients receiving a first index diagnosis of ATPD (F23, ICD-10) within SLaM were included in the study, with a mean follow-up of 1495 days. The overall 8-year risk of developing any non-organic ICD-10 persisting psychotic disorders was $46.25 \%$ (95\% CI 44.17-48.37\%). Specifically, the risk of developing ICD-10 schizophrenia-spectrum disorders was $36.14 \%$ (95\% CI 34.09-38.27\%).

Consistent with our recent meta-analytical estimate of prospective diagnostic stability and instability of brief psychotic episodes 


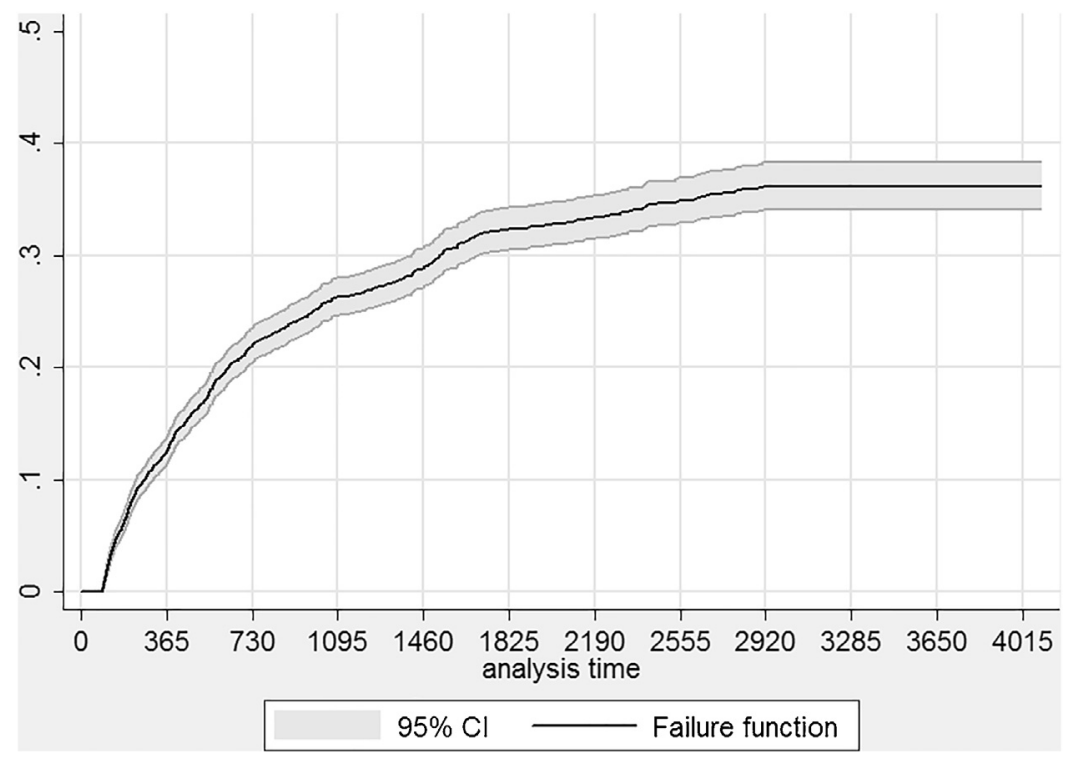

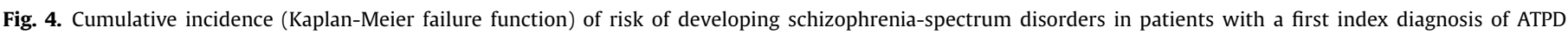
$(n=3074)$.

(ATPD/BPD) [13], the present study suggests that a substantial number of patients with a first index diagnosis of ATPD - just under half - would go on to develop persistent psychotic disorders in the longer term, with about one third of them developing schizophreniaspectrum disorders. This result is of clinical interest because the ATPD group is distinguished from other types of psychoses, such as schizophrenia-spectrum psychoses (Schizophrenia, F20; Schizoaffective disorder, F25; Persistent delusional disorders, F22) on the basis of prototypical symptoms, temporal criteria and early remission [4]. In fact, longitudinal studies have indirectly validated the initial separation of ATPD from frank schizophrenic psychoses. In our recent meta-analysis, we found that approximately half of ATPD patients were still in remission and free from any psychotic relapse (46\%) at more than 3-years. In contrast, only a tiny minority of patients receiving an initial diagnosis of first-episode schizophrenia, and who have experienced a clinical remission (such as for ATPDs), do not relapse (16\%) [2].

In our study, we found a mean time to any non-organic psychotic disorders from ATPD of 1.8 years. We found that patients presenting with schizophrenic symptoms during the index episode of ATPD had a slightly higher risk of developing persistent psychotic disorders, and specifically schizophrenia-spectrum psychoses. These results could provide support to the announced diagnostic revision planned in the upcoming ICD manual (ICD-11), where only the ATPD polymorphic subtype without symptoms of schizophrenia (F23.0) will be retained in the ATPD rubric, while the ATPD subtypes with symptoms of schizophrenia (F23.1 and F23.2) will be collapsed into "unspecified primary psychotic disorders" if lasting less than 4 weeks, and the delusional subtype (F23.3) will be moved to the new category of delusional disorders [31]. Nonetheless, our results are to be interpreted cautiously, as the clinical significance of the slight difference we observed between the two groups requires further clarification. It is also worth noting that the majority of our sample (71\%) received a diagnosis of the residual subtypes - other (F23.8) and unspecified (F23.9) acute and transient psychotic disorders - which suggests that, as previously indicated by others [32], the typical ATPD syndromes - polymorphic, schizophrenia-like, or predominantly delusional - are of difficult applicability in common clinical settings.

Our study may have some clinical implications. We confirmed that patients receiving an ATPD diagnosis have a very high risk of developing persistent psychotic disorders, in particular schizophrenia-spectrum psychoses. Despite their level of psychosis risk being comparable to that of other individuals under the care of CHR-P services, ATPD patients are not systematically detected and signposted for the specialized preventive assessment and treatment which is offered to CHR-P individuals. In a recent observational study conducted in our NHS Trust, we observed that the vast majority of individuals receiving an ATPD diagnosis were not referred to the local CHR-P service [27]. This occurred in spite of an extensive outreach campaign for the detection of individuals at risk of psychosis being promoted and fully implemented by the local CHR-P service. Astonishingly, CHR-P individuals accounted for only $5 \%$ of first-episode psychosis cases, and ATPDs for $26 \%$ of them. It is therefore apparent that preventing the onset of psychotic disorders in ATPD patients has the potential to improve outcomes of first-episode psychosis to a greater extent than focusing on CHR-P individuals only. The additional problem is that currently, there are no specific treatment guidelines for ATPD patients. For example, according to the NICE clinical guideline CG178 "psychosis and schizophrenia in adults: prevention and management" [33], ATPD patients should be offered oral antipsychotic medication in conjunction with psychological therapies. Such a recommendation conflicts with the fact that at the time of their diagnosis, all of these patients would be -by definition- free from any psychotic symptoms. There is no indication as to for how long to continue such a hypothetical antipsychotic treatment, and no clear rationale. For example, no consideration is given to the risk of exposing false positives (about half) to unnecessary antipsychotic treatment. There are also no antipsychotic molecules formally licensed for ATPDs and very few trials have been conducted in these patients. Some evidence suggests that excessive prescription of antipsychotics may actually increase the risk of developing persistent psychotic disorders (such as schizophrenia) in these patients [34]. Although it is argued that firstepisode services may take care of these individuals as for other psychotic diagnoses, there are no robust data showing that ATPD patients remain under the care of these services [35]. Rather, in a time of scarce resources in the NHS, it is more likely that these individuals would be discharged back to their general practitioners soon after their symptoms have resolved. Overall, these 
considerations expose a major window of unmet need. There is also a clear window of missed preventive opportunities. The available alternative could be to detect and signpost ATPD patients for a psychosis risk assessment, such as those performed with validated psychometric tools in CHR-P clinics. For those testing positive, clinical monitoring along with recommended CHR-P preventive interventions could be offered. Such an approach holds the potential to address most of the above limitations, offering an individualized package of care for those ATPD cases most at risk of developing persistent psychotic disorders. Clearly, the first barrier towards this would be our ability -or lack thereof- to detect most of the ATPD patients diagnosed by clinicians [12]. Extensive screening is not sustainable on a financial and logistical scale. However, a recent individualized clinically based risk calculator for the transdiagnostic detection of individuals at risk of developing psychosis (including ATPD cases) has been validated for this purpose [27]. Further research is ongoing and it is hoped that in the near future, we will be better able to detect patients with ATPDs accessing secondary mental health care in order to offer preventive interventions and improve their long-term outcomes [12].

The present study has some limitations, particularly those inherent to the use of electronic health records. We employed the diagnoses recorded in CRIS by clinicians as part of standard clinical practice. Although very good in terms of ecological validity (i.e., they represent real-world clinical practice), they have not been validated with research-based criteria. However, data recorded in electronic health registers has previously been shown to have good reliability in the realm of psychotic categories [36-38]. Moreover, we did not systematically assess patients during the follow-up period, relying instead on data from routine clinical care, which is influenced by a complex interaction of patient and service-related factors. It is possible that patients were discharged from mental health services during the follow-up period because of symptomatic improvement, disengagement from mental health services, moving outside the SLaM catchment area, or other reasons. However, as previously noted in at-risk samples [39], the use of structured diagnostic interviews can lead to selection of patient subsamples and introduce additional biases. Recently we have pragmatically validated our approach, by showing that psychotic outcomes that were estimated through our electronic case register [26] were consistent with those reported by other sites $[10,40]$.

\section{Conclusions}

This study confirmed that approximately half of patients with a first index diagnosis of ATPD will develop a persistent non-organic psychotic disorder over 8-year follow-up. In particular, 36\% of patients with a first ATPD presentation will develop a disorder in the schizophrenia spectrum. ATPDs share diagnostic and prognostic commonalities with other risk syndromes that are currently employed to detect individuals at risk and prevent psychosis. Patients with a first ATPD episode should be referred for a psychosis risk assessment and signposted for preventive interventions.

\section{Declaration of interest}

The authors have no conflicts of interest.

\section{References}

[1] Pillmann F., Marneros A. Brief and acute psychoses: the development of concepts. Hist Psychiatry 2003;14:161-77.

[2] Fusar-Poli P, Cappucciati M, Bonoldi I, Hui LM, Rutigliano G, Stahl DR, et al. Prognosis of brief psychotic episodes: a meta-analysis. JAMA Psychiatry 2016;73:211-20.
[3] Castagnini AC, Fusar-Poli P. Diagnostic validity of ICD-10 acute and transient psychotic disorders and DSM-5 brief psychotic disorder. Eur Psychiatry 2017;45:104-13.

[4] World Health Organization. International Statistical Classification of Diseases. Geneva, Switzerland: World Health Organization; 1992 10th Revision.

[5] American Psychiatric Association. Diagnostic and Statistical Manual of Mental Disorders. 5th ed. Arlington, VA: American Psychiatric Association; 2013.

[6] Fusar-Poli P. The clinical high-risk state for psychosis (CHR-P), version II. Schizophr Bull 2017;43:44-7.

[7] Fusar-Poli P, Rutigliano G, Stahl D, Davies C, De Micheli A, Ramella-Cravaro V, et al. Long-term validity of the At Risk Mental State (ARMS) for predicting psychotic and non-psychotic mental disorders. Eur Psychiatry 2017;42:49-54.

[8] Fusar-Poli P, Cappucciati M, Rutigliano G, Lee TY, Beverly Q, Bonoldi I, et al Towards a standard psychometric diagnostic interview for subjects at ultra high risk of psychosis: CAARMS versus SIPS. Psychiatry J 2016;2016:7146341.

[9] Fusar-Poli P, Cappucciati M, Borgwardt S, Woods SW, Addington J, Nelson B, et al. Heterogeneity of psychosis risk within individuals at clinical high risk: a meta-analytical stratification. JAMA Psychiatry 2016;73:113-20.

[10] Fusar-Poli P, Cappucciati M, Rutigliano G, Schultze-Lutter F, Bonoldi I, Borgwardt S, et al. At risk or not at risk? A meta-analysis of the prognostic accuracy of psychometric interviews for psychosis prediction. World Psychiatry 2015;14:322-32.

[11] Fusar-Poli P, Cappucciati M, De Micheli A, Rutigliano G, Bonoldi I, Tognin S, et al. Diagnostic and prognostic significance of brief limited intermittent psychotic symptoms (BLIPS) in individuals at ultra high risk. Schizophr Bull 2017;43:48-56.

[12] Fusar-Poli P. Extending the benefits of indicated prevention to improve outcomes of first-episode psychosis. JAMA Psychiatry 2017;74:667-8.

[13] Fusar-Poli P, Cappucciati M, Rutigliano G, Heslin M, Stahl D, Brittenden Z, et al. Diagnostic stability of ICD/DSM first episode psychosis diagnoses: metaanalysis. Schizophr Bull 2016;42:1395-406.

[14] Pillmann F, Wustmann T, Marneros A. Acute and transient psychotic disorders versus persistent delusional disorders: a comparative longitudinal study. Psychiatry Clin Neurosci 2012;66:44-52.

[15] Abe T, Otsuka K, Kato S. Long-term clinical course of patients with acute polymorphic psychotic disorder without symptoms of schizophrenia. Psychiatry Clin Neurosci 2006;60:452-7.

[16] Jorgensen P, Bennedsen B, Christensen J, Hyllested A. Acute and transient psychotic disorder: a 1-year follow-up study. Acta Psychiatr Scand 1997;96:150-4.

[17] Suda K, Hayashi N, Hiraga M. Predicting features of later development of schizophrenia among patients with acute and transient psychotic disorder. Psychiatry Clin Neurosci 2005;59:146-50.

[18] Moller HJ, Jager M, Riedel M, Obermeier M, Strauss A, Bottlender R. The Munich 15-year follow-up study (MUFUSSAD) on first-hospitalized patients with schizophrenic or affective disorders: assessing courses, types and time stability of diagnostic classification. Eur Psychiatry 2011;26:231-43.

[19] Remberk B, Bazynska AK, Krempa-Kowalewska A, Rybakowski F. Adolescent insanity revisited: course and outcome in early-onset schizophrenia spectrum psychoses in an 8-year follow-up study. Compr Psychiatry 2014;55:1174-81.

[20] Heslin M, Lomas B, Lappin JM, Donoghue K, Reininghaus U, Onyejiaka A, et al. Diagnostic change 10 years after a first episode of psychosis. Psychol Med 2015:45:2757-69.

[21] Castagnini A, Foldager L. Epidemiology, course and outcome of acute polymorphic psychotic disorder: implications for ICD-11. Psychopathology 2014:47:202-6.

[22] Stewart R, Soremekun M, Perera G, Broadbent M, Callard F, Denis M, et al. The South London and Maudsley NHS Foundation Trust Biomedical Research Centre (SLAM BRC) case register: development and descriptive data. BMC Psychiatry 2009:9:51.

[23] Perera G, Broadbent M, Callard F, Chang CK, Downs J, Dutta R, et al. Cohort profile of the South London and Maudsley NHS Foundation Trust Biomedical Research Centre (SLaM BRC) case register: current status and recent enhancement of an electronic mental health record-derived data resource. BMJ Open 2016;6:e008721.

[24] Roberts E, Wessely S, Chalder T, Chang CK, Hotopf M. Mortality of people with chronic fatigue syndrome: a retrospective cohort study in England and Wales from the South London and Maudsley NHS Foundation Trust Biomedical Research Centre (SLaM BRC) Clinical Record Interactive Search (CRIS) Register: Lancet 2016;387:1638-43.

[25] Oram S, Khondoker M, Abas M, Broadbent M, Howard LM. Characteristics of trafficked adults and children with severe mental illness: a historical cohort study. Lancet Psychiatry 2015;2:1084-91.

[26] Fusar-Poli P, Rutigliano G, Stahl D, Schmidt A, Ramella-Cravaro V, Hitesh S, et al. Deconstructing pretest risk enrichment to optimize prediction of psychosis in individuals at clinical high risk. JAMA Psychiatry 2016;73:1260-7.

[27] Fusar-Poli P, Rutigliano G, Stahl D, Davies C, Bonoldi I, Reilly T, et al. Development and validation of a clinically based risk calculator for the transdiagnostic prediction of psychosis. JAMA Psychiatry 2017;74:493-500.

[28] Benchimol EI, Smeeth L, Guttmann A, Harron K, Moher D, Petersen I, et al. The Reporting of studies conducted using observational routinely-collected health data (RECORD) statement. PLoS Med 2015;12:e1001885.

[29] Kaplan E, Meier P. Nonparametric estimation from incomplete observations. J Am Stat Assoc 1958;53:457-81.

[30] Greenwood M. The Natural Duration of Cancer. London, UK: Her Majesty's Stationery Office; 1926. 
[31] Gaebel W. Status of psychotic disorders in ICD-11. Schizophr Bull 2012;38:895-8

[32] Castagnini A, Bertelsen A, Berrios GE. Incidence and diagnostic stability of ICD-10 acute and transient psychotic disorders. Compr Psychiatry 2008;49:255-61.

[33] NICE. Psychosis and schizophrenia in children and young people: recognition and management. UK, 2014

[34] Wang HY, Guo WJ, Li XJ, Tao YJ, Meng YJ, Wang Q, et al. Higher required dosage of antipsychotics to relieve the symptoms of first-onset Acute and Transient Psychotic Disorder (ATPD) predicted the subsequent diagnostic transition to schizophrenia: a longitudinal study. Schizophr Res 2018;193(Mar)461-2, doi: http://dx.doi.org/10.1016/j.schres.2017.07.011 Epub 2017 Jul 21.

[35] Fusar-Poli P, McGorry PD, Kane JM. Improving outcomes of first-episode psychosis: an overview. World Pychiatry 2017;16:251-65.

[36] Davis KA, Sudlow CL, Hotopf M. Can mental health diagnoses in administrative data be used for research? A systematic review of the accuracy of routinely collected diagnoses. BMC Psychiatry 2016;16:263.
[37] Pihlajamaa J, Suvisaari J, Henriksson M, Heila H, Karjalainen E, Koskela J, et al. The validity of schizophrenia diagnosis in the Finnish Hospital Discharge Register: findings from a 10-year birth cohort sample. Nord J Psychiatry 2008:62:198-203.

[38] Uggerby P, Ostergaard SD, Roge R, Correll CU, Nielsen J. The validity of the schizophrenia diagnosis in the Danish Psychiatric Central Research Register is good. Dan Med J 2013;60:A4578.

[39] Webb JR, Addington J, Perkins DO, Bearden CE, Cadenhead KS, Cannon TD, et al. Specificity of incident diagnostic outcomes in patients at clinical high risk for psychosis. Schizophr Bull 2015;41:1066-75.

[40] Fusar-Poli P, Schultze-Lutter F, Cappucciati M, Rutigliano G, Bonoldi I, Stahl D, et al. The dark side of the moon: meta-analytical impact of recruitment strategies on risk enrichment in the clinical high risk state for psychosis. Schizophr Bull 2016;42:732-43. 